\title{
Ubiquitin-Specific Protease 22/Silent Information Regulator 1 Axis Plays a Pivotal Role in the Prognosis and 5-Fluorouracil Resistance in Hepatocellular Carcinoma
}

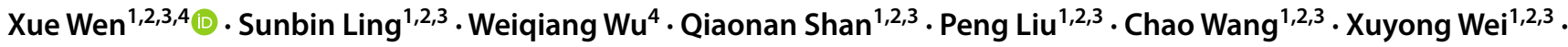

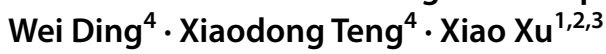

Received: 3 July 2019 / Accepted: 11 September 2019 / Published online: 5 October 2019

(c) The Author(s) 2019

\begin{abstract}
Background Ubiquitin-specific protease 22 (USP22) is described as a key subunit of the Spt-Ada-Gcn5 acetyl transferase complex, which plays an important role in the prognosis and resistance to chemotherapy drugs in hepatocellular carcinoma (HCC). Silent information regulator 1 (SIRT1) is a member of the sirtuin family that is deubiquitinated by USP22. However, it is still unknown whether USP22 and SIRT1 co-expression is associated with disease progression and 5-Fluorouracil (5-FU) resistance in HCC.

Methods 141 patients who received hepatectomy at our hospital from January 2010 to December 2014 were enrolled in this study. The expression of USP22 and SIRT1 was detected by immunohistochemical staining. Clinicopathological features, including age, gender, tumor number, tumor size, tumor differentiation, tumor stage, alpha-fetoprotein and microscopic vascular invasion, were assessed. Further experiments confirmed the role of SIRT1 in 5-FU drug resistance in vivo.

Results Immunohistochemical staining showed that the high expression of USP22 and SIRT1 was frequently observed in HCC tissues relative to normal liver tissues. Overexpression of USP22 is associated with microscopic vascular invasion (MVI). Further analysis showed that the co-expression of USP22 and SIRT1 was more effective in predicting the prognosis of HCC. The SIRT1 inhibitor EX-527 dramatically inhibited the expression of Cyclin B1 and resistance-associated protein 3 (MRP3) to reduce 5-FU drug resistance in vivo.

Conclusion These findings suggest that the co-expression of USP22 and SIRT1 is significantly associated with unfavorable HCC progression. The inhibition of SIRT1 in vivo could be valuable in improving 5-FU drug sensitivity and inhibiting tumor cell proliferation and inducing apoptosis.
\end{abstract}

Keywords Hepatocellular carcinoma $\cdot$ SIRT1 $\cdot$ USP22 $\cdot 5$-Fluorouracil $\cdot$ Drug resistance

Xue Wen, Sunbin Ling and Weiqiang Wu have contributed equally to this work.

\section{Xiao Xu}

zjxu@zju.edu.cn

1 Division of Hepatobiliary and Pancreatic Surgery, Department of Surgery, Collaborative Innovation Center for Diagnosis and Treatment of Infectious Diseases, The First Affiliated Hospital, Zhejiang University School of Medicine, 79 Qingchun Road, Hangzhou 310003, China

2 Key Laboratory of Combined Multi-organ Transplantation, Key Laboratory of Organ Transplantation, Ministry of Public Health, Hangzhou, China
3 Key Laboratory of Organ Transplantation, Hangzhou, Zhejiang, China

4 Department of Pathology, The First Affiliated Hospital, School of Medicine, Zhejiang University, Hangzhou, Zhejiang, China 


\section{Introduction}

Liver cancer is the sixth most common cancer and ranks as the fourth leading cause of cancer-related death. Hepatocellular carcinoma (HCC) accounts for the majority of primary liver tumors [1]. Despite therapeutic advances, the overall survival rate is low due to frequent recurrence and poor responses to both traditional chemotherapy and molecular targeted therapy [2-4]. Therefore, it is urgent to identify target molecules that regulate the biological characteristics of HCC. USP22 is an important subunit of the SAGA (transcriptional cofactor complex), which removes ubiquitin from target proteins to regulate the transcription of downstream genes [5]. In humans, USP22 expression is associated with tumor recurrence, metastasis and poor survival in various cancers [6, 7]. Our group first demonstrated that knockdown of USP22 induces cell cycle arrest and inhibits cell growth in the HCC cell line HepG2 [8].

Silent information regulator 1 (SIRT1), a member of the sirtuin family of nicotinamide adenine dinucleotide $(\mathrm{NAD}+)$-dependent class III histone deacetylases, controls a variety of biologic processes ranging from metabolic homeostasis, neurodegenerative diseases, aging and cancer [9]. Studies have demonstrated that SIRT1 localizes predominantly to the nucleus, and its expression is increased in colorectal [10], ovarian [11] and lung cancers [12]. Xiong et al. [13] demonstrated that SIRT1 is overexpressed in HCC and plays an oncogenic role in HCC by enhancing cell proliferation and resistance to chemotherapy. Interestingly, SIRT1 was also identified as a mediator of acetylation of USP22 and the SAGA coactivator complex [9]. USP22 may decrease levels of p53 through SIRT1 stabilization to suppress cell apoptosis during DNA damage and during embryonic development [14]. In our previous study, USP22 bound to SIRT1 and subsequently activated the AKT pathway, increasing the expression of MRP1 to induce 5-FU resistance in HCC cells [15].

In the current study, we demonstrate, for the first time, that there is a correlation between USP22 and SIRT1 expression and that co-expression of USP22 and SIRT1 may be more effective in predicting prognosis of HCC. However, due to the lack of inhibitors of USP22, we speculate that inhibition of SIRT1 can also inhibit tumor progression and drug resistance. Therefore, we used the SIRT1 inhibitor, EX-527, to analyze whether it can affect 5-FU drug sensitivity.

\section{Materials and Methods}

\section{Patient Samples}

Specimens were obtained from 141 patients with HCC who were surgically treated at our hospital (First Affiliated Hospital, Zhejiang University School of Medicine, Zhejiang, China) between 2010 and 2014. Informed consent was obtained from the patients, and the study was approved by the ethics committee of the First Affiliated Hospital of Zhejiang University. For the normal liver tissue, all cases were selected and tissue was collected at a distance of $>3 \mathrm{~cm}$ from the tumor lesions. These patients were diagnosed with HCC either before or after the surgery. The diagnosis was confirmed by histopathological examination according to the criteria of the World Health Organization (WHO). Immunohistochemical scoring and clinical pathological data were analyzed. Immunohistochemical scoring was determined by the consensus of two pathologists who were blinded to the clinicopathological information associated with the specimens. The clinical and pathological data characteristics of these patients, including gender, age, tumor stage, tumor size, tumor number, tumor differentiation, serum $\alpha$-fetoprotein (AFP) and MVI, were assessed. The mean follow-up period was 40.2 months (range, 1-79 months). All patients were followed up with through March 2017.

\section{Reagents and Antibodies}

5-Fluorouracil (2,4-dihydroxy-5-fluoropyrimidine, 5-FU) and the SIRT1 inhibitor EX-527 were purchased from Selleck Chemicals (Houston, TX, USA). EX-527 inhibited SIRT1 in a concentration-dependent manner with an IC50 of $38 \mathrm{nM}$, but it has much lower potency against SIRT2 (IC50, $19.6 \mu \mathrm{M}$ ) or SIRT3 (IC50, $48.7 \mu \mathrm{M}$ ) [16], and therefore, only SIRT1 expression was detected in this study. The following antibodies were used in immunohistochemical staining: USP22, SIRT1, MRP3 and Cyclin B1; all the antibodies were from Abcam (Cambridge, MA, USA).

\section{Immunohistochemical Staining}

For the tumor samples from HCC patients, a tissue microarray was created from the $141 \mathrm{HCC}$ samples. After histopathological examination, each sample was exercised in one core with a 2.0-mm diameter on one tissue array. According to the manufacturer's protocols for the HistostainTM-Plus kit, immunohistochemical staining was conducted. Primary antibodies were incubated at $4{ }^{\circ} \mathrm{C}$ overnight. Protein expression levels were semiquantitatively scored by assessing the intensity of staining ( 0 points; pale/no yellow, 1 point; mild staining, 2 points; moderate staining, 3 points; strong 
staining) and the percentage of positively stained cells of the total cells in each field $(0,<1 \% ; 1,<25 \%, 2,25-50 \% ; 3$, $50-69 \%$; and $4, \geq 70 \%$ ). Protein expression was expressed as the multiplied score, which was calculated as intensity score $\times$ percentage for positive cells $\times 100 \%$. Finally, samples were grouped as low expression (scores $0-3$ ) or high expression (scores 4-12).

\section{In Vivo Assay}

To investigate whether modified SIRT1 level is related to 5-FU drug sensitivity in nude mice bearing tumor cell xenografts, we used five-week-old male athymic nude mice obtained from the Animal Facility of Zhejiang University, this study was approved by the Medical Ethics Committee of the First Affiliated Hospital of Zhejiang University. The mice were housed in a temperature-controlled animal facility and were provided with sterilized food and water. Briefly, $5-9 \times 10^{6}$ BEL-7402-FU cells, which exhibit high expression of USP22, were injected subcutaneously into the right flank of each nude mouse. Mice with tumors were randomized into four groups (5 mice per group): (A) Vehicles, DMSO; (B) 5-FU, $30 \mathrm{mg} / \mathrm{kg}$; (C) EX-527, $10 \mathrm{mg} / \mathrm{kg}$ and (D) $5-\mathrm{FU}, 30 \mathrm{mg} / \mathrm{kg}+\mathrm{EX}-527,10 \mathrm{mg} / \mathrm{kg}$. The treatment groups received intraperitoneal injection three times per week for 3 weeks. Body weight and subcutaneous tumor size were measured every other day. The tumor volume was detected and calculated using the following formula

Volume $=1 / 2\left(\right.$ length $\times$ width $\left.^{2}\right)$.

\section{TUNEL Assay}

The amount of DNA fragmentation was assessed in paraffin-embedded xenograft sections with a thickness of $4 \mu \mathrm{M}$. Apoptotic cells were visualized using a commercially available kit (In Situ Cell Death Detection Kit, fluorescein, Roche, Indianapolis, IN, USA) according to the manufacturer's procedures. Positive TUNEL staining was observed under a fluorescence microscope (TE2000U, Nikon, Tokyo, Japan). TUNEL-positive apoptotic cells versus total cells were counted in at least five randomly chosen microscopic fields (magnification 200x).

\section{Statistical Analysis}

SPSS 22.0 statistical software and GraphPad Prism software version 6.0 (GraphPad Software, Inc.) were used for statistical analysis. $P$ values for differences between groups were determined by $\chi^{2}$ tests. The overall survival (OS) time was calculated from the day of surgery to the date of mortality or the last follow-up. Survival curves were calculated according to the Kaplan-Meier method. All statistical analyses were performed using paired two-sided $t$ test or one-way analysis of variance (ANOVA). $P<0.05$ was considered a statistically significant difference.

\section{Results}

\section{USP22 and SIRT1 Expression in HCC Tissues and Its Correlation with Clinicopathological Parameters}

Immunohistochemical analyses were performed to detect the expression of USP22 and SIRT1 in tumoral and peri-tumoral tissues. USP22 and SIRT1 were highly expressed in tumoral tissues. By contrast, the expression of USP22 and SIRT1 was weaker in peri-tumoral tissues $\left(\chi^{2}\right.$ test, $P<0.05$, Fig. 1a, c). IHC staining showed that 92 out of the 141 HCC tissues (65.2\%) had high USP22 levels (staining score $>3$, Fig. 1b), all exhibiting positive expression in their nuclei. Similar to USP22 expression, 76 (53.9\%) samples showed high expression of SIRT1 (staining score $>3$, Fig. 1d) in their nuclei. Next, we compared USP22 expression or SIRT1 expression with clinicopathological features, including age, gender, tumor size, tumor number, tumor differentiation, tumor stage, AFP level and MVI in 141 HCC cases (Table 1). Notably, high expression of USP22 was significantly correlated with MVI $\left(\chi^{2}\right.$ test, $\left.P<0.05\right)$. SIRT1 expression was also significantly correlated with tumor differentiation $\left(\chi^{2}\right.$ test, $P<0.001$ ). Thus, high expression of USP22 and SIRT1 was frequently observed and well correlated with malignant behavior in $\mathrm{HCC}$ cases.

\section{Correlation Between USP22 and SIRT1 Expression in HCC}

We further explored the prognostic value of USP22 and SIRT1 expression in patients with HCC. As shown in Fig. 2, the overall survival of patients in the high USP22 group was significantly worse than that of patients in the low USP22 group ( $P=0.000$, Fig. $2 \mathrm{a}$ ), with mean survival times of 37.4 and 69.6 months, respectively. However, no obvious overall survival benefit was found in the SIRT1 group $(P=0.283$, Fig. 2b). High expression of USP22 was significantly correlated with MVI, and the Kaplan-Meier result showed that the overall survival of patients with MVI was poor $(P=0.0019$, log-rank test, Fig. $2 c)$ Next, we evaluated the association of USP22 and SIRT1 expression patterns in HCC samples with the prognosis of patients. These patients were divided into three groups according to the expression patterns of USP22: SIRT1: low expression of all two indicators (USP22-SIRT1-group), high expression of only one indicator (only USP22+ or SIRT1+ group) and high expression of both indicators (USP22+SIRT1+ group). Survival analysis showed that overall survival of patients with co-expression of USP22 and SIRT1 was significantly shorter than overall 

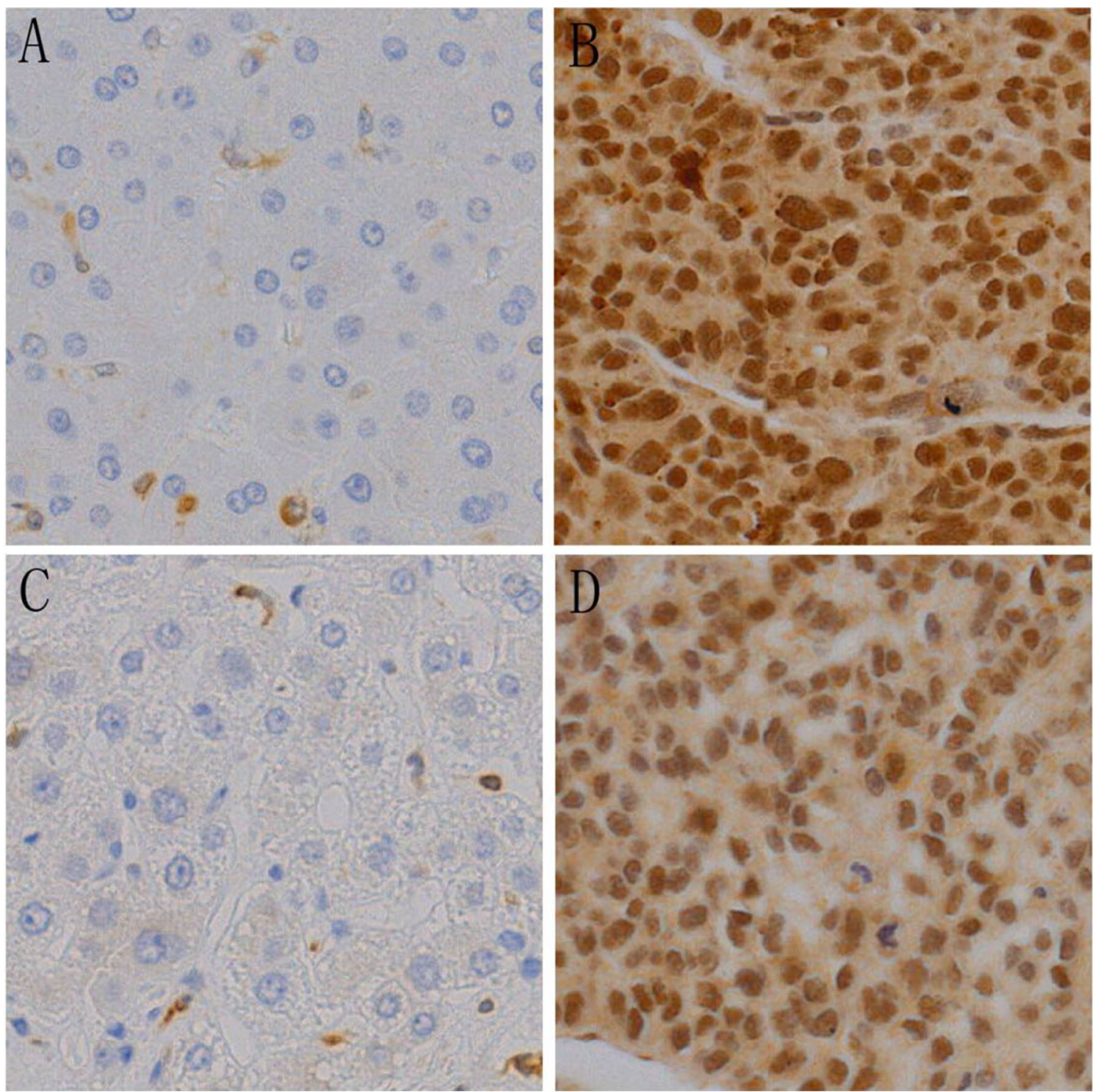

Fig. 1 Immunohistochemical staining for USP22 and SIRT1 in normal tissues and HCC. a USP22 in paracancerous tissues. b USP22 in cancer tissues. c SIRT1 in paracancerous tissues. d SIRT1 in cancer tissues. Magnification, 400×

survival of those with low USP22 or SIRT1 expression and those with low co-expression of both USP22 and SIRT1 $(P=0.003$, Fig. $2 \mathrm{~d})$. Multivariate analysis showed that different USP22 and SIRT1 expression patterns in HCC tissues were an independent prognostic factor, especially in the USP22+SIRT1+ group, where the overall survival was poor. Tumor stage was also an independent prognostic factor for HCC patients undergoing liver resection $(P<0.05$, Table 2$)$. Thus, we demonstrated that USP22 expression was correlated with the overall survival of patients in $\mathrm{HCC}$, and the survival of patients with co-expression of USP22 and SIRT1 was significantly shorter than that of the other groups.

\section{Anti-tumor Activity of 5-FU Alone or in Combination with SIRT1 Inhibitor EX-527 in HCC CDX Models}

Previous research showed that USP22 overexpression in BEL7402-FU cells was related to 5-FU drug resistance [15]. We tested whether the inhibition of SIRT1 by SIRT1 inhibitor EX-527 efficiently changed 5-FU-induced drug resistance in BEL7402-FU cells. We evaluated the anti-tumor activity of 5-FU alone or in combination with SIRT1 inhibitor EX-527 in vivo. No significant difference between the 5-FU single-agent treatment and the control group treated with DMSO $\left(268.8 \pm 65.94 \mathrm{~mm}^{3}\right.$ vs. $559.6 \pm 154.3 \mathrm{~mm}^{3}$, respectively, $P>0.05$, Fig. $\left.3 \mathrm{a}, \mathrm{b}\right)$ was observed. However, compared with the control group, a 
Table 1 Association of USP22 and SIRT1 expression and clinicopathological data of patients with hepatocellular carcinoma

\begin{tabular}{|c|c|c|c|c|c|c|c|}
\hline \multirow[t]{2}{*}{ Clinicopathological } & \multirow[t]{2}{*}{ Cases } & \multicolumn{3}{|c|}{ USP2 2 expression, $n$} & \multicolumn{3}{|c|}{ SIRT1 expression, $n$} \\
\hline & & High & Low & $P^{\alpha}$ & High & Low & $P$ \\
\hline Paracancerous tissues & 141 & 44 & 97 & 0.000 & 17 & 124 & 0.000 \\
\hline $\mathrm{HCC}$ & 141 & 92 & 49 & & 76 & 65 & \\
\hline \multicolumn{8}{|l|}{ Age (years) } \\
\hline$<50$ & 33 & 19 & 14 & 0.290 & 17 & 16 & 0.476 \\
\hline$\geq 50$ & 108 & 73 & 35 & & 48 & 60 & \\
\hline \multicolumn{8}{|l|}{ Sex } \\
\hline Man & 124 & 78 & 46 & 0.114 & 55 & 69 & 0.262 \\
\hline Female & 17 & 14 & 3 & & 10 & 7 & \\
\hline \multicolumn{8}{|l|}{ Tumor number } \\
\hline Single & 135 & 87 & 48 & 0.342 & 60 & 75 & 0.062 \\
\hline Multifocality & 6 & 5 & 1 & & 5 & 1 & \\
\hline \multicolumn{8}{|l|}{ Tumor differentiation } \\
\hline Well & 5 & 2 & 3 & 0.467 & 0 & 5 & 0.000 \\
\hline Well/moderate, moderate & 76 & 51 & 25 & & 25 & 51 & \\
\hline Poor & 60 & 39 & 21 & & 40 & 20 & \\
\hline \multicolumn{8}{|l|}{ Tumor stage } \\
\hline $\mathrm{I}+\mathrm{II}$ & 94 & 59 & 33 & 0.177 & 44 & 50 & 0.811 \\
\hline $\mathrm{III}+\mathrm{IV}$ & 47 & 35 & 14 & & 21 & 26 & \\
\hline \multicolumn{8}{|l|}{$A F P(\mathrm{ng} / \mathrm{ml})$} \\
\hline$<400$ & 8 & 6 & 2 & 0.324 & 3 & 5 & 0.121 \\
\hline$\geq 400$ & 53 & 30 & 23 & & 35 & 18 & \\
\hline \multicolumn{8}{|l|}{ Tumor size $(5 \mathrm{~cm})$} \\
\hline$<5$ & 24 & 17 & 7 & 0.528 & 8 & 16 & 0.168 \\
\hline$\geq 5$ & 117 & 75 & 42 & & 57 & 60 & \\
\hline \multicolumn{8}{|l|}{$M V I$} \\
\hline Negative & 105 & 62 & 43 & 0.008 & 46 & 59 & 0.352 \\
\hline Positive & 36 & 30 & 6 & & 19 & 17 & \\
\hline
\end{tabular}

$\alpha=0.05$, the two-tailed test level of significance

$P<0.05$ was considered a statistically significant difference significantly stronger anti-tumor effect was observed in the combined therapy group $\left(559.6 \pm 154.3 \mathrm{~mm}^{3}\right.$ vs. $92.57 \pm 24.53 \mathrm{~mm}^{3}$, respectively, $P<0.05$ Fig. $3 \mathrm{a}, \mathrm{b}$ ). Tumor sizes and weights in the combination group of both models were significantly decreased compared with those values in the control group (Fig. 3c, d). All treatments were well tolerated. These findings indicated that in high expression of USP22 cells inhibited SIRT1 can improve 5-FU drug resistance.

\section{Inhibition of SIRT1 Effectively Inhibits Tumor Growth and Promotes Cell Apoptosis In Vivo}

Immunohistochemical staining revealed a marked reduction in SIRT1, Cyclin B1, MRP3 and USP22, specifically in xenografts treated with EX-527 in combination with 5-FU (Fig. 4h, 1, p, t). To further analyze whether 5-FU sensitization was a result of increased apoptosis, we performed TUNEL assays to assess the percentage of apoptotic tumor cells in different treatment groups. Compared with vehicle controls, significantly greater TUNEL staining was found in HCC xenografts treated with 5-FU in combination with EX-527 (Fig. 5a, b). These results indicated that anti-SIRT1 therapy reverts resistance and enhances the efficacy of 5-FU in HCC xenografts with acquired 5-FU resistance by increasing apoptosis.

\section{Discussion}

Ubiquitin-specific protease 22 (USP22) is a member of the deubiquitinating enzyme family that can remove ubiquitin from target proteins to regulate the transcription of downstream genes related to tumorigenicity and proliferation [17]. Here, we found that $65.2 \%$ of the $141 \mathrm{HCC}$ cases showed high expression of USP22 by immunohistochemistry. Notably, high expression of USP22 was significantly associated with prognosis in HCC cases. These results were consistent 

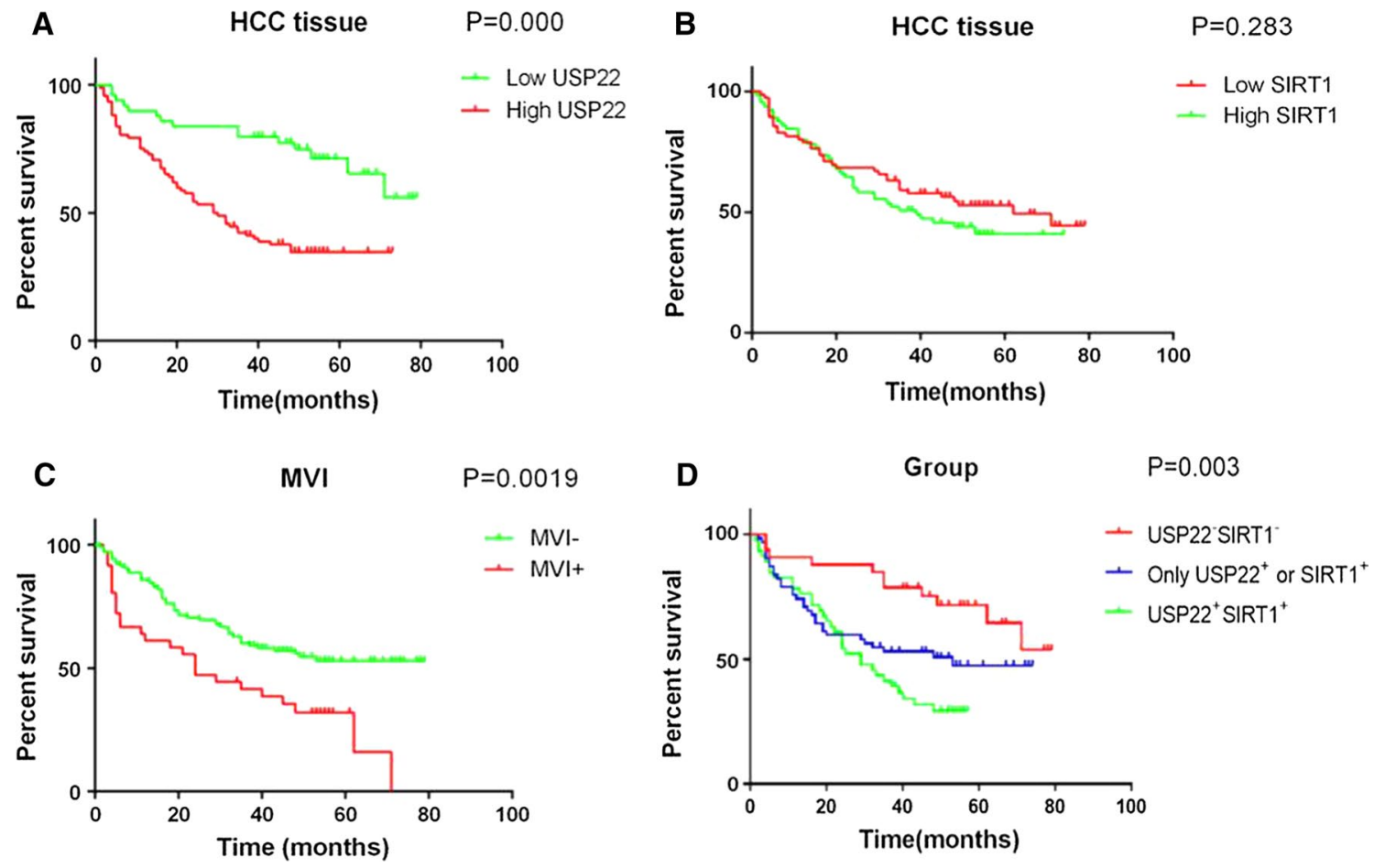

Fig. 2 Kaplan-Meier survival curves of postoperative HCC patients stratified by USP22 and SIRT1 expression. a, b Overall survival curves for HCC patients with regard to USP22 and SIRT1 level in

Table 2 Cox regression analysis of independent prognostic factors in patients with HCC $(n=141)$

\begin{tabular}{lllll}
\hline Variables & $P$ & Hazard ratio & \multicolumn{2}{l}{$\begin{array}{l}\text { Hazard ratio } \\
(95 \% \mathrm{CI})\end{array}$} \\
\cline { 4 - 5 } & & & Lower & Upper \\
\hline $\begin{array}{l}\text { Tumor size }(<5 \mathrm{~cm}, \\
\quad 05 \mathrm{~cm})\end{array}$ & 0.304 & 1.492 & 0.695 & 3.203 \\
$\quad$ Tumor stage & 0.000 & 2.796 & 1.736 & 4.504 \\
MVI & 0.081 & 1.544 & 0.948 & 2.514 \\
Group & 0.009 & & & \\
USP22+SIRT1-group & 0.045 & 2.046 & 1.015 & 4.125 \\
USP22+SIRT1+group & 0.002 & 3.006 & 1.479 & 6.107 \\
\hline
\end{tabular}

$P<0.05$ was considered a statistically significant difference

with the previous literature $[18,19]$. Therefore, USP22 may be a strong prognostic marker and may be a useful therapeutic target for HCC.

As a key subunit of the Spt-Ada-Gen5 acetyl transferase complex (SAGA), USP22 participates in the deubiquitination of NAD-dependent protein deacetylase SIRT1, and USP22 has a pivotal role in antagonizing p53 and stabilizing SIRT1 to suppress cell apoptosis during conditions of DNA damage and during embryonic development $[9,14]$.

tumoral tissues. $\mathbf{c}$ The relation of MVI to survival is shown. d A significant difference among the survival curves in different group of $\mathrm{HCC}$

SIRT1 is overexpressed in hepatocellular carcinoma (HCC) and associated with tumorigenesis, metastasis, prognosis and chemical resistance [20]. In this study, the immunohistochemistry results showed that USP22 and SIRT1 were highly expressed in HCC tissue. Kaplan-Meier analysis showed that high co-expression of USP22 and SIRT1 was associated with shorter OS. Multivariate analysis showed that groups based on the expression of USP22 and SIRT1 patterns in HCC tissues were independent prognostic factors for HCC patients undergoing liver resection. This result is also consistent with our previous finding that USP22 might deubiquitinate SIRT1 in HCC cells [15]. In summary, the USP22/SIRT1 axis may be a useful therapeutic target for HCC. However, due to the lack of inhibitors of USP22, we speculated that inhibition of SIRT1 can also inhibit tumor progression and drug resistance.

The SIRT1 inhibitor EX-527 increased the acetyl-p53/ p53 ratio and FoxO1 acetylation levels, probably exacerbating its ability to induce apoptosis, and reduced the expression of P-gp and MRP3 [21]. Thus, EX-527 could be used during HCC treatment to favor the uptake of a chemotherapeutic agent in cells. Importantly, we observed that the tumor sizes of subcutaneous xenografts was smaller and tumor weights were decreased in the combined 5-FU and EX-527 inhibition group in nude mice bearing tumor 


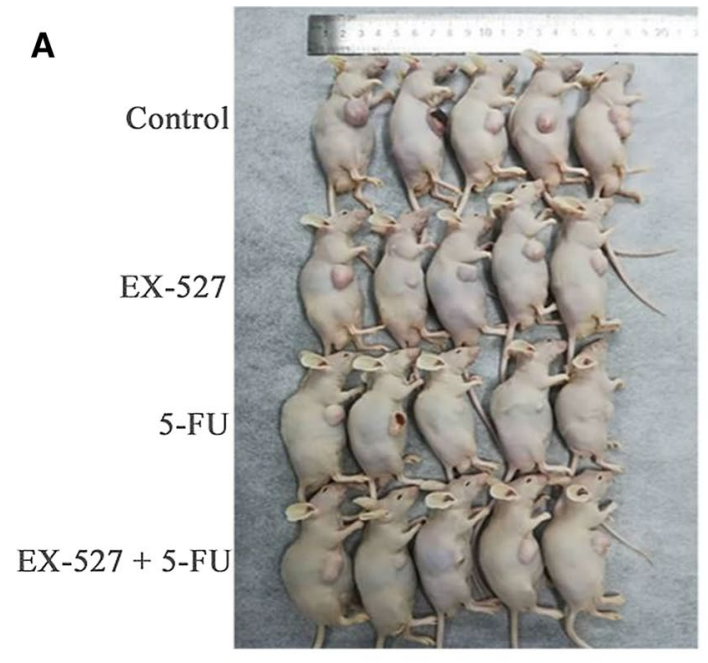

B
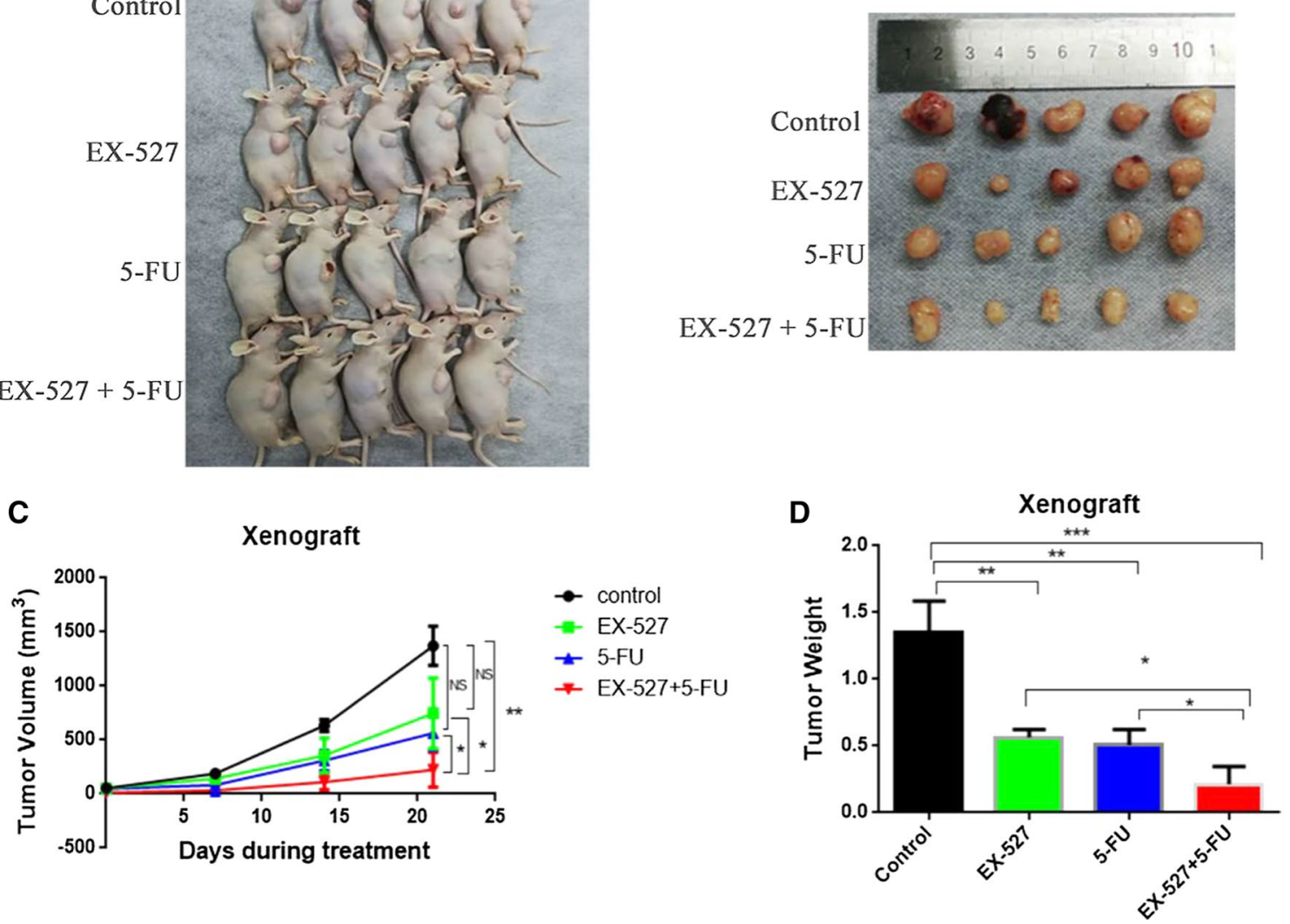

Fig. 3 Mice (5 mice/group) were treated with DMSO, EX-527, 5-FU alone or in combination. Subcutaneous injection of BEL-7402-FU (5-FU-resistant cells with USP22 overexpression) in nude mice (a) and ex vivo images of resected tumors (b). c The xenograft tumor volumes were measured. Each line represents the average tumor vol-

Bel7402-FU cell xenografts. However, there was no significant difference between the 5-FU single-agent treatment group and the control group treated with DMSO. These results indicate that inhibition of SIRT1 can also inhibit tumor growth and reverse USP22 overexpression inducing 5-FU drug resistance in vivo. Interestingly, USP22 levels are significantly upregulated in human brain glioma cells, and it is associated with cell regulatory protein Cyclin B1 expression [22]. Cyclin B1 plays an important role in cell cycle arrest at the $\mathrm{G} 2 / \mathrm{M}$ phase and serves a crucial role in the expansion of tumor cells in HCC [23]. Our results demonstrated that Cyclin B1 expression was decreased in the combined group. On the other hand, USP22 is ume $\left(\mathrm{mm}^{3}\right)$ of each treatment group \pm SD. d Endpoint tumor weights of xenografts. Each line represents the average tumor weight $(\mathrm{g})$ of each treatment group \pm SD. $* P<0.05 ; * * P<0.01 ; * * * P=0.0018 ; N S$ no significant difference

also a stem cell marker that is related to drug resistance [24]. Drug resistance is also an important cause of tumor recurrence and metastasis. Our previous study found that downregulation of USP22 in BEL/FU cells decreases the expression of MRP1, but not P-gp, to regulate 5-FU resistance [15]. As a major multidrug resistance-associated protein, MPR3 was also downregulated in the combined group. Moreover, TUNEL assays also showed that the percentage of apoptotic tumor cells was increased in the combined group. In conclusion, these results indicate that inhibition of SIRT1 may reverse 5-FU drug resistance by inhibiting cell growth via the activation of the cell cycle and induction of apoptosis. We will evaluate the clinical 

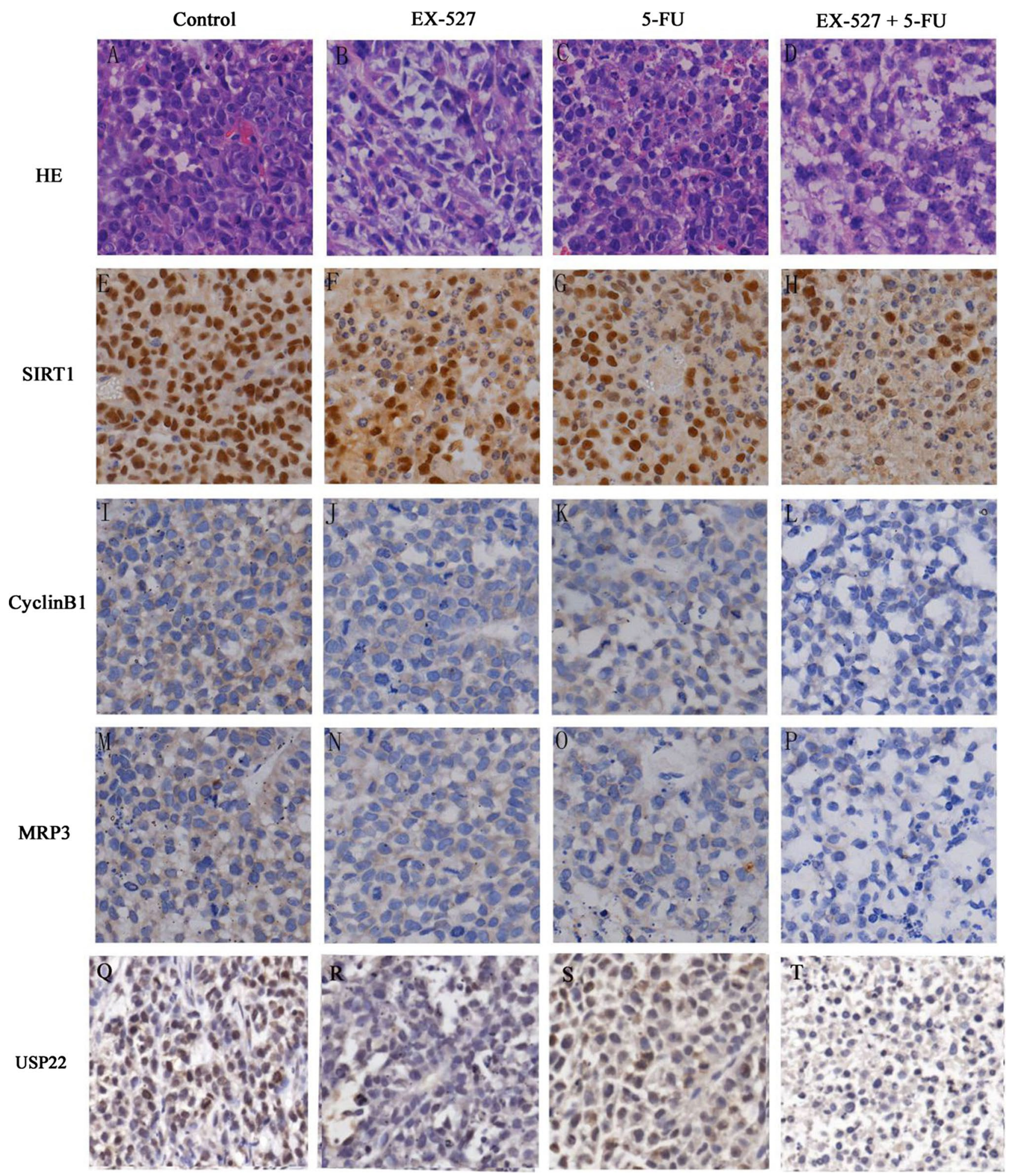

Fig. 4 Subcutaneous injection of BEL-74025-FU-resistant cells in nude mice and ex vivo images of resected tumors. $n=5$ per group. Representative images of a-d H\&E of different groups; $\mathbf{e}-\mathbf{h}$ SIRT1 staining of tumor cells; $\mathbf{i}-\mathbf{l}$ Cyclin B1 positive staining of tumor hepatocytes; m-p MRP3 immunohistochemical staining of resected tumor sections; q-t USP22 immunohistochemical staining of resected tumor sections. Magnification, $400 \times$ 

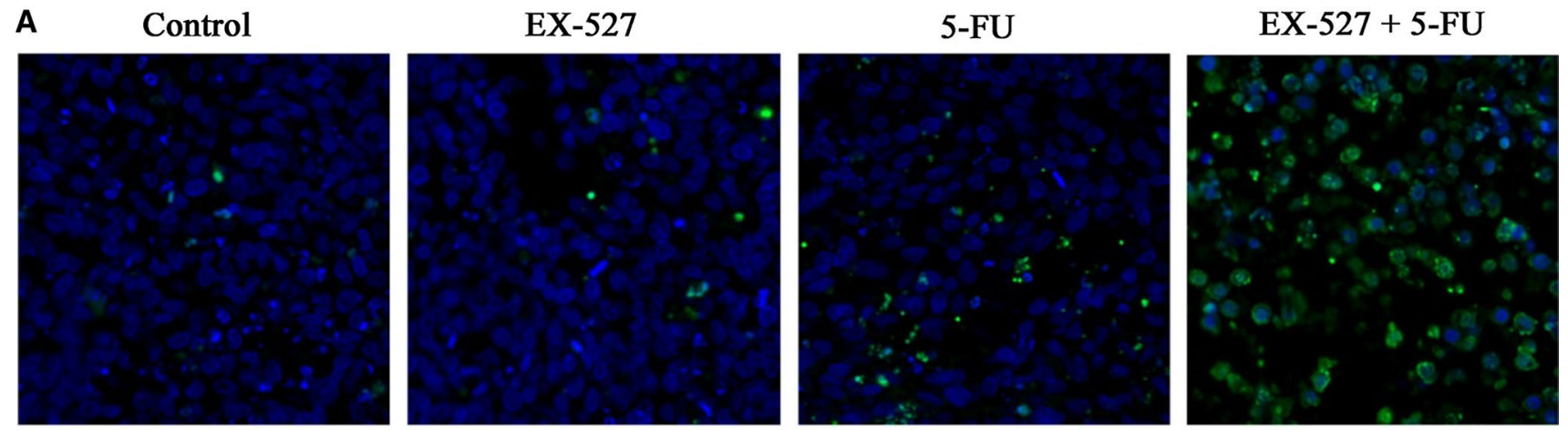

B

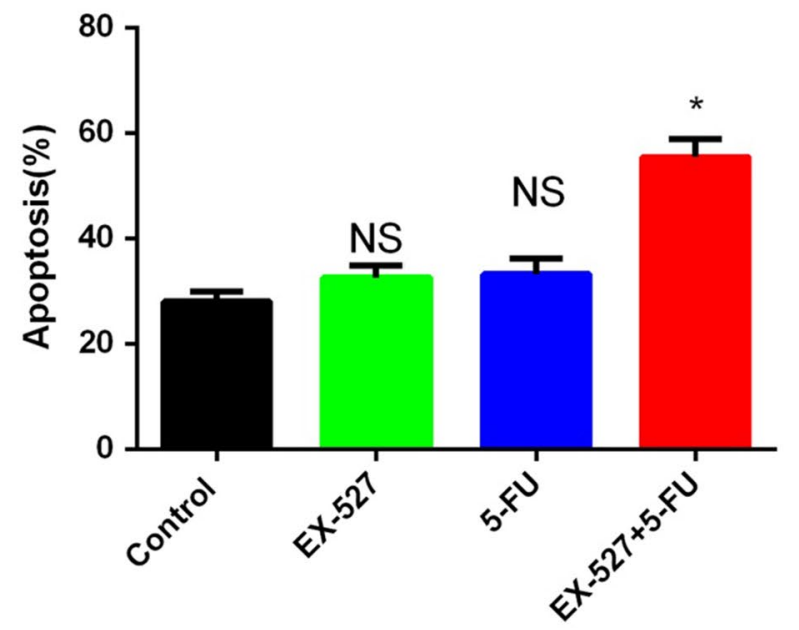

Fig. 5 Representative images of TUNEL-stained (green) sections with DAPI counterstain (blue) for apoptotic cells on tumor tissues harvested from the resected xenografts. Magnification: 200x. Tumor apoptosis was quantified by counting the number of apoptotic cells. Values were expressed as the mean $\pm \mathrm{SD}$. $* P<0.05$ compared to the

significance of USP22, Cyclin B1 and MRP3 expression in HCC tissues for predicting the overall survival or chemotherapy response (Sorafenib or TACE treatment response) in future studies.

\section{Conclusion}

Taken together, the present study found that USP22 and SIRT1 were highly expressed in HCC specimens relative to normal tissue and that expression of both USP22 and SIRT1 was significantly associated with unfavorable HCC characteristics. Furthermore, the presence of the SIRT1 inhibitor EX527 in vivo could also be of great value in identifying therapeutic strategies directed to overcome drug resistance by inhibiting tumor cell proliferation and inducing apoptosis. control group. EX-527 group, $P=0.138$, 5-FU group, $P=0.0074$. Compared to the combined EX-527 and 5-FU group, the $P$ value of EX-527 group and 5-FU group less than 0.05. NS no significant difference

Acknowledgments This research was supported by the National S\&T Major Project (No. 2017ZX10203205), the National Natural Science Foundation of China (No. 81902407) and the Foundation for Zhejiang Medical and Health Science and Technology Project (No. 2018KY376).

Author's contribution XX conceived and coordinated the project. XDT, WD and SBL designed the research study. XW, WQW, CW and QNS performed experiments and acquired data. XYW and PL analyzed and interpreted data. XW and SBL wrote the paper and critically reviewed the manuscript. All authors read and approved the final version of the manuscript.

\section{Compliance with Ethical Standards}

Conflict of interest The authors declare that they have no conflict of interest.

Ethical approval This study was approved by the ethics committee of the First Affiliated Hospital of Zhejiang University and performed 
in accordance with the ethical guidelines denoted in the Declaration of Helsinki (version 2013). Since this is a retrospective analysis, the requirement of a written informed consent was waived by the ethics committee.

Open Access This article is distributed under the terms of the Creative Commons Attribution-NonCommercial 4.0 International License (http://creativecommons.org/licenses/by-nc/4.0/), which permits any noncommercial use, distribution, and reproduction in any medium, provided you give appropriate credit to the original author(s) and the source, provide a link to the Creative Commons license, and indicate if changes were made.

\section{References}

1. Bray F, Ferlay J, Soerjomataram I, Siegel RL, Torre LA, Jemal A. Global cancer statistics 2018: GLOBOCAN estimates of incidence and mortality worldwide for 36 cancers in 185 countries. CA Cancer J Clin. 2018;68:394-424.

2. Njei B, Rotman Y, Ditah I, Lim JK. Emerging trends in hepatocellular carcinoma incidence and mortality. Hepatology. 2015;61:191-199.

3. Chiba T, Iwama A, Yokosuka O. Cancer stem cells in hepatocellular carcinoma: therapeutic implications based on stem cell biology. Hepatol Res. 2016;46:50-57.

4. Guo Z, Li LQ, Jiang JH, Ou C, Zeng LX, Xiang BD. Cancer stem cell markers correlate with early recurrence and survival in hepatocellular carcinoma. World J Gastroenterol. 2014;20:2098-2106.

5. Reyes-Turcu FE, Ventii KH, Wilkinson KD. Regulation and cellular roles of ubiquitin-specific deubiquitinating enzymes. Annu Rev Biochem. 2009;78:363-397.

6. Yang X, Zang H, Luo Y, et al. High expression of USP22 predicts poor prognosis and advanced clinicopathological features in solid tumors: a meta-analysis. Onco Targets Ther. 2018;11:3035-3046.

7. Liu T, Liu J, Chen Q, et al. Expression of USP22 and the chromosomal passenger complex is an indicator of malignant progression in oral squamous cell carcinoma. Oncol Lett. 2019;17:2040-2046.

8. Ling SB, Sun DG, Tang B, et al. Knock-down of USP22 by small interfering RNA interference inhibits HepG2 cell proliferation and induces cell cycle arrest. Cell Mol Biol (Noisy-le-grand). 2012;58:L1803-L1808.

9. Armour SM, Bennett EJ, Braun CR, et al. A high-confidence interaction map identifies SIRT1 as a mediator of acetylation of USP22 and the SAGA coactivator complex. Mol Cell Biol. 2013;33:1487-1502.

10. Yu DF, Jiang SJ, Pan ZP, et al. Expression and clinical significance of Sirt1 in colorectal cancer. Oncol Lett. 2016;11:1167-1172.

11. Jang KY, Kim KS, Hwang SH, et al. Expression and prognostic significance of SIRT1 in ovarian epithelial tumours. Pathology. 2009;41:366-371.
12. Lin SY, Peng F. Association of SIRT1 and HMGA1 expression in non-small cell lung cancer. Oncol Lett. 2016;11:782-788.

13. Xiong H, Ni Z, He J, et al. LncRNA HULC triggers autophagy via stabilizing Sirt1 and attenuates the chemosensitivity of HCC cells. Oncogene. 2017;36:3528-3540.

14. Lin Z, Yang H, Kong Q, et al. USP22 antagonizes p53 transcriptional activation by deubiquitinating Sirt1 to suppress cell apoptosis and is required for mouse embryonic development. Mol Cell. 2012;46:484-494.

15. Ling S, Li J, Shan Q, et al. USP22 mediates the multidrug resistance of hepatocellular carcinoma via the SIRT1/AKT/MRP1 signaling pathway. Mol Oncol. 2017;11:682-695.

16. Solomon JM, Pasupuleti R, Xu L, et al. Inhibition of SIRT1 catalytic activity increases p53 acetylation but does not alter cell survival following DNA damage. Mol Cell Biol. 2006;26:28-38.

17. Zhang XY, Pfeiffer HK, Thorne AW, McMahon SB. USP22, an hSAGA subunit and potential cancer stem cell marker, reverses the polycomb-catalyzed ubiquitylation of histone H2A. Cell Cycle. 2008;7:1522-1524.

18. Tang B, Liang X, Tang F, et al. Expression of USP22 and Survivin is an indicator of malignant behavior in hepatocellular carcinoma. Int J Oncol. 2015;47:2208-2216.

19. Tang B, Tang F, Li B, et al. High USP22 expression indicates poor prognosis in hepatocellular carcinoma. Oncotarget. 2015;6:12654-12667.

20. Wu Y, Meng X, Huang C, Li J. Emerging role of silent information regulator 1 (SIRT1) in hepatocellular carcinoma: a potential therapeutic target. Tumour Biol. 2015;36:4063-4074.

21. Ceballos MP, Decandido G, Quiroga AD, et al. Inhibition of sirtuins 1 and 2 impairs cell survival and migration and modulates the expression of P-glycoprotein and MRP3 in hepatocellular carcinoma cell lines. Toxicol Lett. 2018;289:63-74.

22. Li ZH, Yu Y, Du C, Fu H, Wang J, Tian Y. RNA interference-mediated USP22 gene silencing promotes human brain glioma apoptosis and induces cell cycle arrest. Oncol Lett. 2013;5:1290-1294.

23. Shen $\mathrm{Z}, \mathrm{Yu} \mathrm{X}$, Zheng $\mathrm{Y}$, et al. CDCA5 regulates proliferation in hepatocellular carcinoma and has potential as a negative prognostic marker. Onco Targets Ther. 2018;11:891-901.

24. Jiang S, Song C, Gu X, et al. Ubiquitin-Specific peptidase 22 contributes to colorectal cancer Stemness and chemoresistance via Wnt/beta-Catenin pathway. Cell Physiol Biochem. 2018;46:1412-1422.

Publisher's Note Springer Nature remains neutral with regard to jurisdictional claims in published maps and institutional affiliations. 